\title{
Distance and Eccentric sequences to bound the Wiener index, Hosoya polynomial and the average eccentricity in the strong products of graphs
}

\section{Rocío M. Casablanca*, Peter Dankelmann}

\section{A R T I C L E I N F O}

\section{Article history:}

Received 3 October 2017

Received in revised form 15 May 2018

Accepted 5 July 2018

Available online 16 August 2018

\section{Keywords:}

Wiener index

Average distance

Average eccentricity

Wiener polynomial

Hosoya polynomial

Strong product

Distance sequence

Distance distribution

\begin{abstract}
A B S T R A C T
This paper is concerned with the strong product $G \otimes H$ of two graphs, $G$ and $H$, and bounds on the Wiener index, Hosoya polynomial and the average eccentricity in this family of graphs. We first introduce the distance sequence of a connected graph. It is defined as the sequence of the distances between all unordered pairs of vertices. We prove that the distance sequence of any connected graph of given order and size is dominated by the distance sequence of the so-called path-complete graph. This is the main tool to prove general results as, among others, that, if $G$ is a connected graph of given order and size, then the Wiener index of $G \otimes H$, for every fixed connected graph $H$, and the Hosoya polynomial $W(G, x)$, for every $x \in \mathbb{R}$ with $x \geq 1$, are maximised if $G$ is a path-complete graph. We also investigate the average eccentricity of $G \otimes H$. We show that for fixed $H$, and $G$ chosen from among all connected graphs of given order $n$, it is maximised if $G$ is a path of the same order. We also determine a graph $G_{n, \delta}$ of order $n$ and minimum degree $\delta$ such that for every connected graph $G$ of order $n$ and minimum degree $\delta$, the average eccentricity of $G \otimes H$ never exceeds the average eccentricity of $G_{n, \delta} \otimes H$ by more than 3.
\end{abstract}

(c) 2018 Elsevier B.V. All rights reserved.

\section{Introduction}

Distances between the different points of a network are of great importance in the analysis of the network, and so distance measures are important indicators for communication efficiency in a network. In this paper we consider two distance measures: the Wiener index and the average eccentricity. The Wiener index of a connected graph $G$ with vertex set $V$ is defined by

$$
W(G)=\sum_{\{u, v\} \subseteq V} d(u, v),
$$

where $d(u, v)$ denotes the distance between $u$ and $v$ vertices in $G$ and the sum is taken over all unordered pairs of vertices. The Wiener index found numerous applications in chemistry [25] and it is currently much investigated (see for example $[1,14,19,20])$.

The Wiener index is closely related to the average distance $\mu(G)$ of $G$, defined as $\left(\begin{array}{c}|V| \\ 2\end{array}\right)^{-1} W(G)$. The Hosoya polynomial (also called Wiener Polynomial), introduced by Hosoya in [18] is the polynomial $W(G, x)=\sum_{\{u, v\} \subseteq V} x^{d(u, v)}$, which has been and recently studied $[8,9,26,30]$.

\footnotetext{
This research was supported by the Ministry of Economy and Competitiveness, Spain, under project MTM2014-60127-P, the University of Seville, Spain and the University of Johannesburg, South Africa.

* Corresponding author.

E-mail addresses: rociomc@us.es (R.M. Casablanca), pdankelmann@uj.ac.za (P. Dankelmann).
} 
The average eccentricity (see, for example, [6]) is defined as the arithmetic mean of the eccentricities of the vertices,

$$
\operatorname{avec}(G)=|V|^{-1} \sum_{v \in V} \operatorname{ecc}(v),
$$

where the eccentricity ecc $(v)$ of a vertex $v$ is defined as the distance to a vertex farthest from $v$.

Graph products play an important role in the design of networks since they often allow us to build larger versions of a network preserving certain desirable properties. For designing large-scale interconnection networks, the graph products are useful methods to obtain large graphs from smaller ones whose invariants can be easily calculated. For more information on this topic we refer the reader to the book by Hammack, Imrich and Klavžar [16]. We have focused on the strong product of graphs, which is one of the classical graph products, and there is an extensive literature on it. The strong product $G \otimes H$ of two connected graphs $G$ and $H$ is the graph with vertex set $V(G) \times V(H)$ in which two vertices $(a, u)$ and $(b, v)$ are adjacent if $a=b$ and $u v \in E(H)$, or $a b \in E(G)$ and $u=v$, or $a b \in E(G)$ and $u v \in E(H)$. From the definition, it clearly follows that the strong product of two graphs is commutative and that it is connected if and only if both graphs are connected.

While the Wiener index of the cartesian product and some other products of graphs can be expressed relatively easily in terms of the Wiener index and other basic information of the two component graphs (see [32]), expressing the Wiener index of the strong product of graphs seems to be less simple (see $[3,23]$ ). The same holds true for the average eccentricity (see $[7,12])$. Hence it is reasonable to consider bounds on the Wiener index and the average eccentricity of the strong product of graphs.

The following basic result on the Wiener index has been proved independently by Doyle and Graver [10], Lovász [22] and Plesník, [24].

Theorem $\mathbf{1}([10,11,22,24])$. Let $G$ be a connected graph of order $n$. Then

$$
W(G) \leq \frac{n(n+1)(n-1)}{6} .
$$

Equality holds if and only if $G$ is a path.

The starting point of our investigations is a generalisation by Casablanca, Favaron and Kouider [3], showing that for every fixed, connected graph $H$, the Wiener index of $G \otimes H$, where $G$ ranges over all connected graphs of given order $n$, is maximised if $G$ is the path $P_{n}$. The special case $H=K_{1}$ in Theorem 2 is Theorem 1.

Theorem 2 ([3]). Let $H$ be a connected graph. If $G$ is a connected graph of order $n$, then

$$
W(G \otimes H) \leq W\left(P_{n} \otimes H\right),
$$

with equality only if $G$ is a path.

We strengthen this result in different ways. In Section 3 we present some results on the distance sequence of a graph, defined as the non-decreasing sequence of the distances between all unordered pairs of vertices. These form the basis for the following two sections. In Section 4 we generalise Theorem 2, showing that if we prescribe not only the order but also the size of $G$, then for every fixed, connected graph $H$, the Wiener index of $G \otimes H$ is maximised if $G$ is the so-called pathcomplete graph $P K_{n, m}$, defined in Section 2. We also strengthen Theorem 2 for $G$ being a $2 k$-connected graph. In this case $W(G \otimes H)$ is maximised if $G$ is the $k$ th power of a cycle. We also determine graphs $G$ that minimise $W(G \otimes H)$ for every fixed, connected graph $H$ for $G$ being a graph of given order and size, a tree, a planar graph, an outerplanar graph, a graph with no clique $K_{r}$ where $r \in \mathbb{N}$, or a graph of given order and diameter. In Section 5 we apply the results from Section 2 to derive some properties of the Hosoya polynomial $W(G, x)$. We show that every connected graph $G$ and for $x \in \mathbb{R}$ we have $W(G, x) \leq W\left(P_{n}, x\right)$ if $x \geq 1$ and $W(G, x) \geq W\left(P_{n}, x\right)$ for $x \leq 1$. We show similar relations with $P_{n}$ replaced by $P K_{n, m}$ if $G$ has order $n$ and size $m$, with $P_{n}$ replaced by $C_{n}^{\bar{k}}$ if $G$ has order $n$ and is $2 k$-connected. We also show that the opposite inequalities hold for certain graphs. In Section 6 we obtain results for eccentric sequences of graphs, similar to those for the distance sequence in Section 3. In the final section, Section 7 we obtain results for the average eccentricity of $G \otimes H$, similar to those for the Wiener index in Section 4 by determining graphs $G$ that maximise avec $(G \otimes H)$ where $G$ is a graph of given (i) order, (ii) order and (even) connectivity, and (iii) order and minimum degree.

\section{Notation}

Our notation is as follows. If $G$ is a graph, then we denote its vertex and edge set by $V(G)$ and $E(G)$, respectively. We use $n_{G}$ and $m_{G}$ for the order and size of $G$, respectively. If $v$ is a vertex, then $N_{G}(v)$ and $N_{G}[v]$ are the neighbourhood of $v$, i.e., the set of vertices adjacent to $v$, and the closed neighbourhood, i.e., the set $N_{G}(v) \cup\{v\}$, respectively. The distance $d_{G}(u, v)$ between two vertices $u$ and $v$ is the minimum number of edges on a $(u, v)$-path in $G$. The diameter of $G, d(G)$, is the maximum distance between two vertices of $G$. If $v$ is a vertex of $G$ and $k \in \mathbb{N}$, then $N_{k}(v)$ denotes the set of vertices at distance exactly $k$ from $v$. By $N_{\leq k}(v)$ we mean the set $\bigcup_{i=1}^{k} N_{i}(v)$. The distance between a vertex $v$ and a set $W \subseteq V(G)$ is defined as $\min _{w \in W} d_{G}(v, w)$. Let $\operatorname{deg}_{G}(v)$ be the degree of $v$, i.e., the cardinality of $N_{G}(v)$, and $\delta(G)$ is the minimum degree of $G$. If there is no ambiguity we often drop the subscript or argument $G$. 
By $P_{n}, C_{n}, K_{n}$ and $K_{a, b}$ we denote the path, cycle, complete graph and complete bipartite graph with partite sets of cardinality $a$ and $b$, respectively, of order $n$. The graph with $n$ vertices and no edges is denoted by $\overline{K_{n}}$. The $k$ th power $G^{k}$ of a graph $G$ is the graph with vertex set $V(G)$ in which two vertices are adjacent if their distance is not more than $k$. If $G_{1}$ and $G_{2}$ are two graphs, then their sum $G_{1}+G_{2}$ (also called their join) is the graph obtained from their disjoint union by adding edges joining every vertex of $G_{1}$ to every vertex of $G_{2}$.

If $X=\left(x_{1}, \ldots, x_{m}\right)$ and $Y=\left(y_{1}, \ldots, y_{n}\right)$ are two sequences of integers and $c \in \mathbb{N}$, then the sequence $\left(x_{1}, \ldots, x_{m}, y_{1}, \ldots\right.$, $\left.y_{n}\right)$ is the concatenation of $X$ and $Y$, denoted by $X \circ Y$, and $X^{c}$ denotes the sequence obtained from $X$ by repeating every entry $c$ times, $\left(x_{1}, x_{1}, \ldots, x_{1}, x_{2}, x_{2}, \ldots, x_{2}, \ldots, x_{m}, x_{m}, \ldots, x_{m}\right)$, and $c X$ is the sequence $\left(c x_{1}, \ldots, c x_{m}\right)$. If $m=n$, then $X+Y$ is the sequence $\left(x_{1}+y_{1}, \ldots, x_{m}+y_{m}\right)$.

\section{Distance sequences of graphs}

The main tool in our investigations of the Wiener index of the strong product of graphs is the distance sequence, defined below. It is closely related to the distance distribution of a graph, which was introduced by Buckley and Superville [2] and further investigated in $[4,27,31]$.

Definition 1. Let $G$ be a connected graph of order $n$. The distance sequence $\mathcal{D}(G)$ is the non-decreasing sequence $\left(d_{1}, d_{2}, \ldots, d_{\left(\begin{array}{c}n \\ 2\end{array}\right)}\right)$ of the distances between all unordered pairs of distinct vertices of $G$. If $A$ is a set of pairs of vertices of $G$, then $\left.\mathcal{D}(G)\right|_{A}$ denotes the non-decreasing sequence of the distances between all the unordered pairs of vertices in $A$. The distance degree of a vertex $v$ of $G$, denoted by $\mathcal{D}(G, v)$, is the non-decreasing sequence of the distances between $v$ and all other vertices of $G$.

Definition 2. Let $\mathcal{A}=\left(a_{1}, a_{2}, \ldots, a_{k}\right)$ and $\mathcal{B}=\left(b_{1}, b_{2}, \ldots, b_{k}\right)$ be two sequences of the same length. We say that $\mathcal{A}$ dominates $\mathcal{B}$ if $a_{i} \geq b_{i}$ for $i=1,2, \ldots, k$, and we write $\mathcal{A} \geq \mathcal{B}$.

In this section we show that certain graphs that are known to have maximum Wiener index among all graphs of given order in a particular class of graphs possess a much stronger property: their distance sequence dominates, or is dominated by, the distance sequence of every connected graph of the same order within their particular class of graphs. We make extensive use of this property in Sections 4 and 5.

Our first result of this kind shows that the distance sequence of a path-complete graph (defined below) dominates the distance sequence of all connected graphs of the same order and size. Path-complete graphs were introduced by Harary in [17].

Definition 3 ([17]). A graph is path-complete if it can be obtained from the union of a path and a clique by joining one end of the path to at least one vertex of the clique. For given $n, m \in \mathbb{N}$ with $n-1 \leq m \leq\left(\begin{array}{c}n \\ 2\end{array}\right)$ there is a unique path-complete of order $n$ and size $m$, which is denoted by $P K_{n, m}$.

Šoltés [28] showed that the path-complete graph $P K_{n, m}$ has maximum average distance among all connected graphs of order $n$ and size $m$. A simple expression for the Wiener index of $P K_{n, m}$ was given by Gutman and Šoltés in [15]. In his proof, Šoltés made use of a stronger property of path-complete graphs, which we use to show that the distance sequence of $P K_{n, m}$ dominates that of any connected graph of order $n$ and size $m$. For $k \in \mathbb{N}$ define $s_{k}(G)$ to be the number of all unordered pairs of non-adjacent vertices of $G$ at distance at most $k$.

Lemma 1 ([28]). Let $G$ be a connected graph of order $n$, size $m$ and diameter $d$, where $d \geq 3$. Then, for all $k$ with $2 \leq k \leq d-1$,

$$
s_{k}(G) \geq s_{k}\left(P K_{n, m}\right) .
$$

Lemma 2. If $G$ is a connected graph of order $n$ and size $m$, then

$$
\mathcal{D}(G) \leq \mathcal{D}\left(P K_{n, m}\right) .
$$

Proof. Let $d=\operatorname{diam}(G)$ and let $\mathcal{D}(G)=\left(d_{1}, d_{2}, \ldots, d_{\left(\begin{array}{c}n \\ 2\end{array}\right)}\right)$ and $\mathcal{D}\left(P K_{n, m}\right)=\left(d_{1}^{\prime}, d_{2}, \ldots, d_{\left(\begin{array}{c}n \\ 2\end{array}\right)}^{\prime}\right)$. So it suffices to show that

$$
d_{i} \leq d_{i}^{\prime} \text { for } i=1,2, \ldots,\left(\begin{array}{l}
n \\
2
\end{array}\right) .
$$

Suppose to the contrary that there exists $j \in\left\{1,2, \ldots,\left(\begin{array}{l}n \\ 2\end{array}\right)\right\}$ with $d_{j}>d_{j}^{\prime}$. Let $j$ be the smallest number with this property. Since both, $G$ and $P K_{n, m}$ have size $m$, we have $d_{i}=d_{i}^{\prime}=1$ for $i=1,2, \ldots, m$, and thus $j>m$ and $d_{j}^{\prime} \geq 2$. This implies in particular that $d \geq 3$.

It is immediate from the definition of $s_{k}$ that for $k$ with $2 \leq k \leq d-1$,

$$
d_{i}=k \quad \Leftrightarrow \quad m+s_{k-1}(G)+1 \leq i \leq m+s_{k}(G) .
$$


Now let $d_{j}=k$ and $d_{j}^{\prime}=k^{\prime}<k$. From (2) we get

$$
m+s_{k-1}(G)+1 \leq j \leq m+s_{k}(G)
$$

and

$$
m+s_{k^{\prime}-1}\left(P K_{n, m}\right)+1 \leq j \leq m+s_{k^{\prime}}\left(P K_{n, m}\right) .
$$

Comparing the left hand side of the first and the right hand side of the second of these inequalities, we conclude that

$$
s_{k-1}(G)+1 \leq s_{k^{\prime}}\left(P K_{n, m}\right) .
$$

By $k^{\prime} \leq k-1$ and the fact that the sequence $s_{i}\left(P K_{n, m}\right)$ is increasing in $i$, we get

$$
s_{k-1}(G)<s_{k-1}\left(P K_{n, m}\right) \text {. }
$$

Since $2 \leq d_{j}^{\prime}=k^{\prime} \leq k-1$, as observed above, we have a contradiction to Lemma 1 . This proves (1).

Corollary 1. Let $G$ be a connected graph of order $n$. Then

$$
\mathcal{D}(G) \leq \mathcal{D}\left(P_{n}\right)
$$

Proof. Let $T$ be a spanning tree of $G$. Clearly, $\mathcal{D}(G) \leq \mathcal{D}(T)$. Applying Lemma 2 with $m=n-1$ and noting that $P K_{n, n-1}=P_{n}$ we get $\mathcal{D}(T) \leq \mathcal{D}\left(P_{n}\right)$. Combining these two inequalities yields the corollary.

Remark 1. It is natural to ask if it is true that, whenever $G$ and $G^{\prime}$ are connected graphs of the same order, then $\mathcal{D}(G) \leq \mathcal{D}\left(G^{\prime}\right)$ if and only if $W(G) \leq W\left(G^{\prime}\right)$. While clearly $\mathcal{D}(G) \leq \mathcal{D}\left(G^{\prime}\right)$ implies $W(G) \leq W\left(G^{\prime}\right)$, the converse is not true in general. Consider the graphs $F$ and $F^{\prime}$ below:
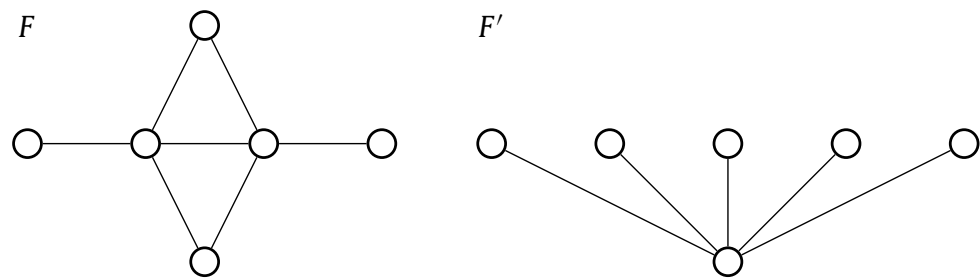

Then $\mathcal{D}(F)=\left(3,2^{7}, 1^{7}\right)$ and $\mathcal{D}\left(F^{\prime}\right)=\left(2^{10}, 1^{5}\right)$, and so $W(F)=24$ and $W\left(F^{\prime}\right)=25$. Clearly, $W(F) \leq W\left(F^{\prime}\right)$ but $\mathcal{D}(F) \not \leq \mathcal{D}\left(F^{\prime}\right)$.

Lemma 3. Let $G$ be a $2 k$-connected graph of order $n$, where $k \in \mathbb{N}$. Then

$$
\mathcal{D}(G) \leq \mathcal{D}\left(C_{n}^{k}\right)
$$

Proof. It suffices to show that for every vertex $v$ of $G$ and every vertex $w$ of $C_{n}^{k}$,

$$
\mathcal{D}(G, v) \leq \mathcal{D}\left(C_{n}^{k}, w\right) .
$$

Let $v$ be an arbitrary vertex of $G$ and let $\mathcal{D}(G, v)=\left(a_{1}, a_{2}, \ldots, a_{n-1}\right)$. Since $G$ is $2 k$-connected, $v$ has at least $2 k$ vertices at distance $i$ for $i=1,2, \ldots, \operatorname{ecc}(v)-1$. It follows that $a_{1}, a_{2}, \ldots, a_{2 k} \leq 1, a_{2 k+1}, a_{2 k+2}, \ldots, a_{4 k} \leq 2, a_{4 k+1}, a_{4 k+2}, \ldots, a_{6 k} \leq 3$ and so on. Hence

$$
\mathcal{D}(G, v) \leq\left(1,2, \ldots,\left\lceil\frac{n-1}{2 k}-1\right\rceil\right)^{2 k} \circ\left(\left\lceil\frac{n-1}{2 k}\right\rceil\right)^{b}
$$

where $b=n-1-2 k\left\lceil\frac{n-1}{2 k}-1\right\rceil$.

Let $w$ be an arbitrary vertex of $C_{n}^{k}$. It is easy to verify that $w$ has exactly $2 k$ vertices at distance $i$ for $i=1,2, \ldots,\left\lceil\frac{n-1}{2 k}-1\right\rceil$ and the remaining vertices have distance $\left\lceil\frac{n-1}{2 k}\right\rceil$. It follows that

$$
\mathcal{D}\left(C_{n}^{k}, w\right)=\left(1,2, \ldots,\left\lceil\frac{n-1}{2 k}-1\right\rceil\right)^{2 k} \circ\left(\left\lceil\frac{n-1}{2 k}\right\rceil\right)^{b},
$$

where $b=n-1-2 k\left\lceil\frac{n-1}{2 k}-1\right\rceil$ as above. Hence $\mathcal{D}(G, v) \leq \mathcal{D}\left(C_{n}^{k}, w\right)$, as desired.

We now present graphs of given order and diameter whose distance sequence is dominated by every graph with the same order and diameter. 
Definition 4. Let $G$ be a graph of order $n$ and diameter $d$. Let $G^{n, d}$ be the graph obtained from the disjoint union of a path $P_{d+1}: v_{0}, v_{1}, \ldots, v_{d}$ and a clique $K_{n-d-1}$ by joining every vertex in the clique to $v_{\lfloor d / 2\rfloor-1}, v_{\lfloor d / 2\rfloor}$ and $v_{\lfloor d / 2\rfloor+1}$.

Lemma 4. Let $G$ be a graph of order $n$ and diameter $d$ and $G^{n, d}$ be the graph described in Definition 4. Then

$$
\mathcal{D}(G) \geq \mathcal{D}\left(G^{n, d}\right) .
$$

Proof. Let $v_{0}$ and $v_{d}$ be two vertices of $G$ at distance $d$, and let $P: v_{0}, v_{1}, \ldots, v_{d}$ be a shortest $\left(v_{0}, v_{d}\right)$-path in $G$. We partition the set of all unordered pairs of vertices of $G$ into three sets, $A, B$ and $C$, where $A$ is the set of pairs of vertices that are both on $P, B$ the set of pairs of vertices that are both not on $P$, and $C$ the set of pairs where one vertex is on $P$ and the other one is not. Clearly,

$$
\left.\mathcal{D}(G)\right|_{A}=\mathcal{D}(P)=\mathcal{D}\left(P_{d+1}\right) .
$$

and

$$
\left.\mathcal{D}(G)\right|_{B} \geq(1)^{\left(\begin{array}{c}
n-d-1 \\
2
\end{array}\right)} \text {. }
$$

We now consider the distances between pairs in $C$. Let $u \in V(G)-V(P)$ and let $k:=d_{G}\left(u, v_{0}\right)$. Then $d_{G}\left(u, v_{k}\right) \geq 1$. Since $d\left(u, v_{i}\right) \geq|k-i|$ for $i=0,1, \ldots, d$, the distances from $u$ to $v_{k-1}, v_{k-2}, \ldots, v_{0}$ are at least $1,2, \ldots, k$, and the distances for $u$ to $v_{k+1}, v_{k+2}, \ldots, v_{d}$ are at least $1,2, \ldots, d-k$. Hence, with $j:=\min (k, n-k)$ and $j^{\prime}:=\max (k, n-k)$, the distances from $u$ to the vertices of $P$ are at least $\left(1,1,1,2,2,3,3, \ldots, j, j, j+1, j+2, \ldots, j^{\prime}-1, j^{\prime}\right)$. Observe that the latter dominates $\left(1,1,1,2,2,3,3, \ldots, \frac{n-2}{2}, \frac{n-2}{2}, \frac{n}{2}\right)$ when $n$ is even, but in the case that $n$ is odd, $\left(1,1,1,2,2,3,3, \ldots, \frac{n-1}{2}, \frac{n-1}{2}\right)$. Hence

$$
\begin{aligned}
\mathcal{D}(G, v) & \geq \begin{cases}\left(1,1,1,2,2,3,3, \ldots, \frac{n-2}{2}, \frac{n-2}{2}, \frac{n}{2}\right) & \text { if } n \text { is even, } \\
\left(1,1,1,2,2,3,3, \ldots, \frac{n-1}{2}, \frac{n-1}{2}\right) & \text { if } n \text { is odd. }\end{cases} \\
& =1 \circ \mathcal{D}\left(P_{d+1}, c\right),
\end{aligned}
$$

where $c$ is a central vertex of the path $P_{d+1}$.

Now for $G^{n, d}$, (3), (4), and (5) hold with equality. Let $A^{\prime}, B^{\prime}$ and $C^{\prime}$ be the set of all unordered pairs of vertices of $G^{n, d}$ that have both vertices are in $P_{d+1}$, both vertices are in $K_{n-d-1}$, and one vertex in $P_{d+1}$ and one vertex in $K_{n-d-1}$, respectively. Hence

$$
\left.\mathcal{D}\left(G^{n, d}\right)\right|_{A^{\prime}} \leq\left.\mathcal{D}(G)\right|_{A},\left.\quad \mathcal{D}\left(G^{n, d}\right)\right|_{B^{\prime}} \leq\left.\mathcal{D}(G)\right|_{B},\left.\quad \mathcal{D}\left(G^{n, d}\right)\right|_{C^{\prime}} \leq\left.\mathcal{D}(G)\right|_{\mathcal{C}}
$$

Hence we conclude that $\mathcal{D}\left(G^{n, d}\right) \leq \mathcal{D}(G)$, as desired.

We conclude this section by showing that among the graphs of given order and size, the distance sequences of those of diameter two are dominated by all such graphs. Applying this result to some classes of graphs, we get that in those classes, a graph that has maximum size relative to its order and also diameter at most two, has a distance sequence that is dominated by every other graph of the same order in this class.

Proposition 1. Let $G$ be a connected graph of order $n$ and size not more than $m$. If $G^{\prime}$ is a graph of order $n$, size $m$, and diameter at most two, then

$$
\mathcal{D}(G) \geq \mathcal{D}\left(G^{\prime}\right),
$$

with equality if and only if $\operatorname{diam}(G) \leq 2$ and $G$ has size $m$.

Proof. Let $\mathcal{D}(G)=\left(d_{1}, d_{2}, \ldots, d_{\left(\begin{array}{c}n \\ 2\end{array}\right)}\right)$. Since $G$ has size not more than $m$, we have $d_{1}, d_{2}, \ldots, d_{m} \geq 1$ and $d_{m+1}, d_{m+2} \ldots, d_{\left(\begin{array}{l}n \\ 2\end{array}\right)}$ $\geq 2$. Hence,

$$
\mathcal{D}(G) \geq(1)^{m} \circ(2)^{\left(\begin{array}{c}
n \\
2
\end{array}\right)-m},
$$

Clearly, equality holds if and only if $\operatorname{diam}(G) \leq 2$ and $G$ has size $m$. The proposition follows.

Corollary 2. Let $G$ be a connected graph of order $n$.

(a) If $G$ is a tree then $\mathcal{D}(G) \geq \mathcal{D}\left(K_{1, n-1}\right)$.

(b) If $G$ is a planar graph then $\mathcal{D}(G) \geq \mathcal{D}\left(K_{2}+P_{n-2}\right)$.

(c) If $G$ is an outerplanar graph then $\mathcal{D}(G) \geq \mathcal{D}\left(K_{1}+P_{n-1}\right)$.

(d) If $G$ has no $k$-clique then $\mathcal{D}(G) \geq \mathcal{D}\left(T_{n, k}\right)$, where $T_{n, k}$ is the Turán graph, i.e., the balanced, complete $(k-1)$-partite graph. 


\section{Bounds on the Wiener index of the strong product}

Several upper and lower bounds on the Wiener index of graphs of given order with certain properties are known. The following upper and lower bounds are relevant for this section. The graphs in these two theorems were defined in Section 3.

Theorem 3. Let $G$ be a connected graph of order $n$.

(a) ([28]) If $G$ has size $m$ then $W(G) \leq W\left(P K_{n, m}\right)$.

(b) ([13]) If $G$ is $2 k$-connected then $W(G) \leq W\left(C_{n}^{k}\right)$.

Theorem 4. Let $G$ be a connected graph of order $n$.

(a) ([24]) If $G$ has diameter $d$, then $W(G) \geq W\left(G^{n, d}\right)$.

(b) ([24]) If $G$ has size $m$ then $W(G) \geq n(n-1)-m$, with equality if $\operatorname{diam}(G) \leq 2$.

(c) ([24]) If $G$ is a tree then $W(G) \geq W\left(K_{1, n-1}\right)$.

(d) ([24]) If $G$ is a planar graph then $W(G) \geq W\left(K_{2}+P_{n-2}\right)$.

(e) ([24]) If $G$ is an outerplanar graph then $W(G) \geq W\left(K_{1}+P_{n-1}\right)$.

(f) ([24]) If $G$ has no $k$-clique then $W(G) \geq W\left(T_{n, k}\right)$.

In this section we use the results of Section 3 to show that the graphs in Theorems 3 and 4 do not only maximise or minimise $W(G)$, but have the stronger property of maximising or minimising the strong product with any connected graph in the same sense as in Theorem 2. The following Lemma allows us to derive these results from the results on distance sequences in the preceding section.

Lemma 5. Let $G$ and $G^{\prime}$ be two connected graphs of the same order. If $\mathcal{D}(G) \geq \mathcal{D}\left(G^{\prime}\right)$ then

$$
W(G \otimes H) \geq W\left(G^{\prime} \otimes H\right)
$$

for every connected graph $H$. If equality holds for some connected graph $H$, then we have $\mathcal{D}(G)=\mathcal{D}\left(G^{\prime}\right)$.

Proof. Let $H, G$ and $G^{\prime}$ be as in the hypothesis of the lemma. Let $n$ be the order of $G$ and $G^{\prime}$, and let $\mathcal{D}(G)=\left(d_{1}, d_{2}, \ldots, d_{\left(\begin{array}{c}n \\ 2\end{array}\right)}\right)$ and $\mathcal{D}\left(G^{\prime}\right)=\left(d_{1}^{\prime}, d_{2}^{\prime}, \ldots, d_{\left(\begin{array}{c}n \\ 2\end{array}\right)}^{\prime}\right)$. Then $d_{i} \geq d_{i}^{\prime}$ for $i=1,2, \ldots,\left(\begin{array}{c}n \\ 2\end{array}\right)$. Making use of the fact that for $a, b \in V(G)$ and $u, v \in V(H)$, we have $d_{G \otimes H}((a, u),(b, v))=\max \left(d_{G}(a, b), d_{H}(u, v)\right)$, we obtain

$$
\begin{aligned}
W(G \otimes H) & =\frac{1}{2} \sum_{a, b \in V(G)} \sum_{u, v \in V(H)} d_{G \otimes H}((a, u),(b, v)) \\
& =\frac{1}{2} \sum_{a, b \in V(G)} \sum_{u, v \in V(H)} \max \left(d_{G}(a, b), d_{H}(u, v)\right) \\
& =n W(H)+\sum_{i=1}^{\left(\begin{array}{c}
n \\
2
\end{array}\right)} \sum_{u, v \in V(H)} \max \left(d_{i}, d_{H}(u, v)\right) \\
& \geq n W(H)+\sum_{i=1}^{\left(\begin{array}{c}
n \\
2
\end{array}\right)} \sum_{u, v \in V(H)} \max \left(d_{i}^{\prime}, d_{H}(u, v)\right) \\
& =\frac{1}{2} \sum_{a, b \in V\left(G^{\prime}\right)} \sum_{u, v \in V(H)} \max \left(d_{G^{\prime}}(a, b), d_{H}(u, v)\right) \\
& =\frac{1}{2} \sum_{a, b \in V\left(G^{\prime}\right)} \sum_{u, v \in V(H)} d_{G^{\prime} \otimes H}((a, u),(b, v)) \\
& =W\left(G^{\prime} \otimes H\right),
\end{aligned}
$$

as desired.

To see that (6) holds note the following. If $(a, u),(b, v)$ are two vertices of $G \otimes H$ with $a \neq b$, then the vertices are in distinct copies of $H$, and so $d_{G \otimes H}((a, u),(b, v))$ appears twice in the sum $\sum_{a, b \in V(G)} \sum_{u, v \in V(H)} \max \left(d_{G}(a, b), d_{H}(u, v)\right)$ but only once in $\sum_{i=1}^{\left(\begin{array}{l}n \\ 2\end{array}\right)} \sum_{u, v \in V(H)} \max \left(d_{i}, d_{H}(u, v)\right)$. If $(a, u)$ and $(b, v)$ are two vertices of $G \otimes H$ with $a=b$, then $d_{G \otimes H}((a, u),(a, v))$ is not counted in $\sum_{i=1}^{\left(\begin{array}{c}n \\ 2\end{array}\right)} \sum_{u, v \in V(H)} \max \left(d_{i}, d_{H}(u, v)\right)$ but in $n W(H)$.

Assume that $W(G \otimes H)=W\left(G^{\prime} \otimes H\right)$ for some connected graph $H$. Then we have equality in (7), and so $\max \left(d_{i}, d_{H}(u, v)\right)=$ $\max \left(d_{i}^{\prime}, d_{H}(u, v)\right)$ for all $u, v \in V(H)$ and all $i$. If $u v \in E(H)$, then $d_{H}(u, v)=1$, and so $d_{i}=d_{i}^{\prime}$ for all $i$. Hence $\mathcal{D}(G)=\mathcal{D}\left(G^{\prime}\right)$, as desired. 
Remark 2. It is natural to ask if it is true that whenever $G$ and $G^{\prime}$ are connected graphs with $W(G) \leq W\left(G^{\prime}\right)$, then $W(G \otimes H) \leq W\left(G^{\prime} \otimes H\right)$ for every connected graph $H$. The graphs $F$ and $F^{\prime}$ defined in Section 3 show that this is not the case.

As observed, we have $W(F)<W\left(F^{\prime}\right)$. Substitute $F$ for $G, F^{\prime}$ for $G^{\prime}$, and $F^{\prime}$ for $H$. But it is easy to check that $\mathcal{D}\left(F \otimes F^{\prime}\right)=$ $\left(3^{36}, 2^{452}, 1^{142}\right)$ and $\mathcal{D}\left(F^{\prime} \otimes F^{\prime}\right)=\left(2^{520}, 1^{110}\right)$ and so $W\left(F \otimes F^{\prime}\right)=1154$ and $W\left(F^{\prime} \otimes F^{\prime}\right)=1150$, implying that $W\left(F \otimes F^{\prime}\right)>$ $W\left(F^{\prime} \otimes F^{\prime}\right)$.

Lemma 5 combined with Lemmas 2-4, Proposition 1 and Corollary 2 yields the following theorems.

Theorem 5. Let $H$ be a connected graph. If $G$ is a connected graph of order $n$ and size $m$, then

$W(G \otimes H) \leq W\left(P K_{n, m} \otimes H\right)$.

The special case $m=n-1$ yields Theorem 2 .

Theorem 6. Let $H$ be a connected graph. If $G$ is a $2 r$-connected graph of order $n$ where $r \in \mathbb{N}$, then $W(G \otimes H) \leq W\left(C_{n}^{r} \otimes H\right)$.

Theorem 7. Let $G$ be a connected graph of order $n$ and diameter $d$ and $G^{n, d}$ be the graph described in Definition 4 . Then, for every connected graph $\mathrm{H}$,

$$
W(G \otimes H) \geq W\left(G^{n, d} \otimes H\right) .
$$

Theorem 8. Let $G$ be a connected graph of order $n$ and size not more than $m$. If $G^{\prime}$ is a graph of order $n$, size $m$, and diameter at most two, then

$$
W(G \otimes H) \geq W\left(G^{\prime} \otimes H\right) .
$$

with equality if and only if $\operatorname{diam}(G) \leq 2$.

Corollary $3(a)$. Let $T$ be a tree of order $n$. Then for every connected graph $H$,

$$
W(T \otimes H) \geq W\left(K_{1, n-1} \otimes H\right) .
$$

(b) Let $G$ be a connected planar graph of order $n$, where $n \geq 5$. Then for every connected graph $H$,

$$
W(G \otimes H) \geq W\left(\left(K_{2}+P_{n-2}\right) \otimes H\right) .
$$

(c) Let $G$ be a connected outerplanar graph of order $n$, where $n \geq 5$. Then for every connected graph $H$,

$$
W(G \otimes H) \geq W\left(\left(K_{1}+P_{n-1}\right) \otimes H\right) .
$$

(d) Let $k \in \mathbb{N}$ with $k \geq 3$. Let $G$ be a connected graph of order $n$ with no complete subgraph of order $k$. Then for every connected graph $H$,

$$
W(G \otimes H) \geq W\left(T_{n, k} \otimes H\right) .
$$

\section{On the Hosoya polynomial of graphs with certain properties}

In this section we apply the results on the distance sequence proved in Section 2 to Hosoya polynomials. The key observation on which the results in this section rest is the following lemma.

Lemma 6. Let $G$ and $G^{\prime}$ be two connected graphs of the same order. If $\mathcal{D}(G) \geq \mathcal{D}\left(G^{\prime}\right)$ then, for $x \in \mathbb{R}$,

$$
\begin{aligned}
& W(G, x) \geq W\left(G^{\prime}, x\right) \text { if } x \geq 1, \\
& W(G, x) \leq W\left(G^{\prime}, x\right) \text { if } 0 \leq x \leq 1 .
\end{aligned}
$$

Proof. Let $G$ and $G^{\prime}$ be connected graphs of order $n$ with distance sequence $\left(d_{1}, d_{2}, \ldots, d_{\left(\begin{array}{c}n \\ 2\end{array}\right)}\right)$ and $\left(d_{1}^{\prime}, d_{2}^{\prime}, \ldots, d_{\left(\begin{array}{l}n \\ 2\end{array}\right)}^{\prime}\right)$, respectively. By the hypothesis of the theorem we have $d_{i} \geq d_{i}^{\prime}$ for $i=1,2, \ldots,\left(\begin{array}{c}n \\ 2\end{array}\right)$. Hence for $x \in \mathbb{R}$,

$$
\begin{aligned}
W(G, x)-W\left(G^{\prime}, x\right) & =\left(n+\sum_{i=1}^{\left(\begin{array}{c}
n \\
2
\end{array}\right)} x^{d_{i}}\right)-\left(n+\sum_{i=1}^{\left(\begin{array}{c}
n \\
2
\end{array}\right)} x^{d_{i}^{\prime}}\right) \\
& =\sum_{i=1}^{\left(\begin{array}{c}
n \\
2
\end{array}\right)}\left(x^{d_{i}}-x^{d_{i}^{\prime}}\right) .
\end{aligned}
$$

Hence, since $d_{i} \geq d_{i}^{\prime}$ we have $x^{d_{i}}-x^{d_{i}^{\prime}} \geq 0$ for $x \geq 1$, and $x^{d_{i}}-x^{d_{i}^{\prime}} \leq 0$ for $0 \leq x \leq 1$, and the lemma follows. 
Applying Lemma 6 to the results in Chapter 2, specifically to Lemmas 2-4, Proposition 1 and Corollaries 1 and 2 we obtain the following results.

Theorem 9. For every connected graph $G$ of order $n$ we have

$$
\begin{aligned}
& W(G, x) \leq W\left(P_{n}, x\right) \text { for all } x \in \mathbb{R} \text { with } x \geq 1, \\
& W(G, x) \geq W\left(P_{n}, x\right) \text { for all } x \in \mathbb{R} \text { with } 0 \leq x \leq 1 .
\end{aligned}
$$

Theorem 10. For every connected graph $G$ of order $n$ and size $m$ we have

$$
\begin{aligned}
& W(G, x) \leq W\left(P K_{n, m}, x\right) \quad \text { for all } x \in \mathbb{R} \text { with } x \geq 1, \\
& W(G, x) \geq W\left(P K_{n, m}, x\right) \text { for all } x \in \mathbb{R} \text { with } 0 \leq x \leq 1 .
\end{aligned}
$$

Theorem 11. For every $2 k$-connected graph $G$ of order $n$ we have

$$
\begin{aligned}
& W(G, x) \leq W\left(C_{n}^{k}, x\right) \quad \text { for all } x \in \mathbb{R} \text { with } x \geq 1, \\
& W(G, x) \geq W\left(C_{n}^{k}, x\right) \text { for all } x \in \mathbb{R} \text { with } 0 \leq x \leq 1 .
\end{aligned}
$$

Theorem 12. If $G^{n, d}$ is the graph described in Definition 4 , for every connected graph $G$ of order $n$ and diameter $d$ we have

$$
\begin{aligned}
& W(G, x) \geq W\left(G^{n, d}, x\right) \quad \text { for all } x \in \mathbb{R} \text { with } x \geq 1, \\
& W(G, x) \leq W\left(G^{n, d}, x\right) \quad \text { for all } x \in \mathbb{R} \text { with } 0 \leq x \leq 1 .
\end{aligned}
$$

Theorem 13. Let $G$ be a connected graph of order $n$ and size at least $m$, and let $G^{\prime}$ be a connected graph of order $n$, size $m$, and diameter at most two. Then

$$
\begin{aligned}
& W(G, x) \geq W\left(G^{\prime}, x\right) \quad \text { for all } x \in \mathbb{R} \text { with } x \geq 1, \\
& W(G, x) \leq W\left(G^{\prime}, x\right) \text { for all } x \in \mathbb{R} \text { with } 0 \leq x \leq 1 .
\end{aligned}
$$

Theorem $14(a)$. Let $G$ be a tree of order $n$. Then

$$
\begin{aligned}
& W(G, x) \geq W\left(K_{1, n-1}, x\right) \text { for all } x \in \mathbb{R} \text { with } x \geq 1, \\
& W(G, x) \leq W\left(K_{1, n-1}, x\right) \text { for all } x \in \mathbb{R} \text { with } 0 \leq x \leq 1 .
\end{aligned}
$$

(b) Let $G$ be a connected planar graph of order $n$. Then

$$
\begin{aligned}
& W(G, x) \geq W\left(K_{2}+P_{n-2}, x\right) \quad \text { for all } x \in \mathbb{R} \text { with } x \geq 1, \\
& W(G, x) \leq W\left(K_{2}+P_{n-2}, x\right) \text { for all } x \in \mathbb{R} \text { with } 0 \leq x \leq 1 .
\end{aligned}
$$

(c) Let $G$ be a connected outerplanar graph of order $n$. Then

$$
\begin{aligned}
& W(G, x) \geq W\left(K_{1}+P_{n-1}, x\right) \quad \text { for all } x \in \mathbb{R} \text { with } x \geq 1, \\
& W(G, x) \leq W\left(K_{1}+P_{n-1}, x\right) \quad \text { for all } x \in \mathbb{R} \text { with } 0 \leq x \leq 1 .
\end{aligned}
$$

(d) Let $G$ be a connected graph of order $n$ with no complete subgraph of order $k$. Then

$$
\begin{aligned}
& W(G, x) \geq W\left(T_{n, k}, x\right) \text { for all } x \in \mathbb{R} \text { with } x \geq 1, \\
& W(G, x) \leq W\left(T_{n, k}, x\right) \quad \text { for all } x \in \mathbb{R} \text { with } 0 \leq x \leq 1 .
\end{aligned}
$$

We note that essentially the same proof yields similar statements for the $k$ th derivative of the Hosoya polynomial and all $x \in \mathbb{R}$ with $x \geq 1$. 


\section{On eccentric sequences of graphs}

In this section we consider eccentric sequences of graphs, introduced by Lesniak [21] and our aim is to prove results on the eccentric sequence similar to those in Section 3 on the distance sequence. Recall that the eccentricity of a vertex $v$ in a connected graph $G$ is defined as the distance from $v$ to a vertex farthest from $v$.

Definition 5. Let $G$ be a connected graph of order $n$. The eccentric sequence $\mathcal{E}(G)$ is the non-increasing sequence $\left(e_{1}, e_{2}, \ldots, e_{n}\right)$ of the eccentricities of the vertices of $G$. If $A \subseteq V(G)$ is a set of vertices of $G$, then $\left.\mathcal{E}(G)\right|_{A}$ is the non-increasing sequence of the eccentricities in $G$ of the vertices in $A$.

Lesniak [21] characterised sequences that are eccentric sequences of trees. The problem of finding a characterisation of eccentric sequences of graphs seems hard, and a solution remains elusive. To date, the only graph class other than trees for which a characterisation of their eccentric sequences is known are maximal outerplanar graphs (see [5]). The following result shows that eccentric sequence of any connected graph is dominated by the eccentric sequence of a path, when both graphs have the same order.

Lemma 7. Let $G$ be a connected graph of order $n$. Then

$$
\mathcal{E}(G) \leq \mathcal{E}\left(P_{n}\right) .
$$

Proof. Let $\left(e_{1}, e_{2}, \ldots, e_{n}\right)$ be the eccentric sequence of a connected graph $G$ of order $n$. We show that

$$
e_{i} \leq n-\left\lceil\frac{i}{2}\right\rceil \text { for } i=1,2, \ldots, n \text {. }
$$

Let $T$ be a spanning tree of $G$. It suffices to prove (8) for T since the eccentricity of a vertex in a spanning tree of $G$ is not less than its eccentricity in $G$.

The proof is by induction on $n$. Inequality (8) clearly holds for $n=2$ and $n=3$. Now let $n \geq 4$. Let $u$ and $v$ be two vertices of $T$ at distance $\operatorname{diam}(T)$, and let $T^{\prime}=T-\{u, v\}$. Since $u$ and $v$ are end-vertices of $T$, the graph $T^{\prime}$ is connected and thus a tree. Let $e_{1}^{*}, e_{2}^{*}, \ldots, e_{n-2}^{*}$ be the eccentric sequence of $T^{\prime}$. By our induction hypothesis we have $e_{i}^{*} \leq n-2-\left\lceil\frac{i}{2}\right\rceil$ for $i=1,2, \ldots, n-2$. The eccentricity of $u$ and $v$ is at most $n-1$, and removing $u$ and $v$ has reduced the eccentricities of the remaining vertices of $T$ by at most one. Hence

$$
e_{1} \leq n-1, e_{2} \leq n-1
$$

and

$$
e_{i} \leq e_{i-2}^{*}+1 \leq n-2-\left\lceil\frac{i-2}{2}\right\rceil+1=n-\left\lceil\frac{i}{2}\right\rceil,
$$

for $i=1, \ldots, n$, which implies (8).

It is easy to verify that if $G=P_{n}$, then we have equality in (8) for all $i$. Hence the lemma follows.

Lemma 8. Let $G$ be a $2 k$-connected graph of order $n$, where $k \in \mathbb{N}$. Then

$$
\mathcal{E}(G) \leq \mathcal{E}\left(C_{n}^{k}\right)
$$

Proof. Since $G$ is $2 k$-connected, the eccentricity of any vertex cannot exceed $\left\lceil\frac{n-1}{2 k}\right\rceil$. Hence

$$
\mathcal{E}(G) \leq\left(\left\lceil\frac{n-1}{2 k}\right\rceil,\left\lceil\frac{n-1}{2 k}\right\rceil, \ldots,\left\lceil\frac{n-1}{2 k}\right\rceil\right) .
$$

It is easy to verify that

$$
\mathcal{E}\left(C_{n}^{k}\right)=\left(\left\lceil\frac{n-1}{2 k}\right\rceil,\left\lceil\frac{n-1}{2 k}\right\rceil, \ldots,\left\lceil\frac{n-1}{2 k}\right\rceil\right) .
$$

Hence the Lemma follows.

We now consider graphs of given order and minimum degree $\delta$. Below we define a graph $G_{n, \delta}$ whose eccentric sequence 'almost dominates' that of every connected graph of order $n$ and minimum degree $\delta$. More precisely, we show that no term in the eccentric sequence of a graph of order $n$ and minimum degree $\delta$ exceeds the corresponding term in $\mathcal{E}\left(G_{n, \delta}\right)$ by more than 3.

Example 1. Consider the graph $P_{\ell, \delta}$, obtained from a path $P_{3 \ell}: v_{0}, v_{1}, \ldots, v_{3 \ell-1}$ by adding sets $V_{i}$ of $\delta-2$ new vertices which form a clique and are adjacent to $v_{i-1}, v_{i}$ and $v_{i+1}$ for $i=1,4,7, \ldots, 3 \ell-2$. Fig. 1 shows $P_{5,4}$. 


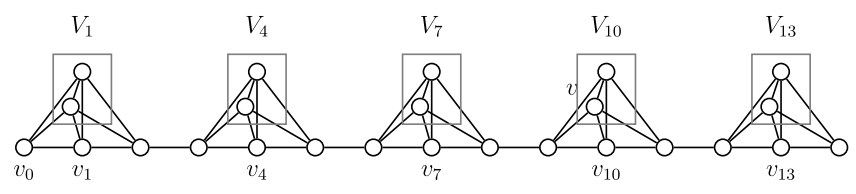

Fig. 1. The graph $P_{5,4}$.

If $n \geq \ell(\delta+1)+2$ then let $G_{n, \delta}^{(\ell)}$ be the graph obtained from $P_{\ell, \delta}$ by adding a vertex adjacent to all vertices in $\left\{v_{0}, v_{1}, v_{2}\right\} \cup V_{1}$, and a clique on $n-\ell(\delta+1)-1$ vertices, all adjacent to the vertices in $\left\{v_{3 \ell-3}, v_{3 \ell-2}, v_{3 \ell-1}\right\} \cup V_{3 \ell-2}$. We now determine the eccentric sequence of $G_{n, \delta}^{(\ell)}$. Let $A=\left\{v_{1}, v_{4}, v_{7}, \ldots, v_{3 \ell-2}\right\}$. Then it is easy to verify that

$$
\left.\mathcal{E}\left(P_{\ell, \delta}\right)\right|_{A}=3 \mathcal{E}\left(P_{\ell}\right)+(1)^{\ell} .
$$

Let $B_{i}=N\left[v_{3 i-2}\right]$ for $i=1,2, \ldots, \ell$. Then $\left|B_{i}\right|=\delta+1$ and $\left|\operatorname{ecc}(v)-\operatorname{ecc}\left(v_{3 i-2}\right)\right| \leq 1$ for $i=1,2, \ldots, \ell$, and $V\left(G_{n, \delta}^{\ell}\right)$ is the disjoint union of the $B_{i}$. Hence

$$
\mathcal{E}\left(P_{\ell, \delta}\right)=\left(3 \mathcal{E}\left(P_{\ell}\right)+(1)^{\ell}\right)^{\delta+1}+\epsilon_{\ell(\delta+1)},
$$

where $\epsilon_{\ell(\delta+1)}$ is a sequence of length $\ell(\delta+1)$ whose entries are in $\{-1,0,1\}$. Since the vertices added to $P_{\ell, \delta}$ to obtain $G_{n, \delta}^{(\ell)}$ have eccentricity $3 \ell-1$ we get

$$
\mathcal{E}\left(G_{n, \delta}^{(\ell)}\right)=(3 \ell-1)^{n-\ell(\delta+1)} \circ\left(\left(3 \mathcal{E}\left(P_{\ell}\right)+(1)^{\ell}\right)^{\delta+1}+\epsilon_{\ell(\delta+1)}\right) .
$$

It is easy to see that if $\ell_{1} \leq \ell_{2}$ and $n \geq \ell_{1}(\delta+1)+2$, then $\mathcal{E}\left(G_{n, \delta}^{\left(\ell_{1}\right)}\right) \leq \mathcal{E}\left(G_{n, \delta}^{\left(\ell_{2}\right)}\right)$. We define the graph $G_{n, \delta}$ as $\mathcal{E}\left(G_{n, \delta}^{(\ell)}\right)$ with the largest possible value for $\ell$, i.e., with $\ell=\left\lfloor\frac{n-2}{\delta+1}\right\rfloor$. Then

$$
\mathcal{E}\left(G_{n, \delta}^{(\ell)}\right) \leq \mathcal{E}\left(G_{n, \delta}\right)
$$

Lemma 9. Let $G$ be a connected graph of order $n$ and minimum degree $\delta$. Then

$$
\mathcal{E}(G) \leq \mathcal{E}\left(G_{n, \delta}\right)+(3)^{n},
$$

where $G_{n, \delta}$ is as defined in Example 1.

Proof. We first construct a maximal packing $A$ of $G$ as follows. Choose a vertex $a_{1}$ of $G$. If $G$ contains a vertex at distance exactly three from $\left\{a_{1}\right\}$ then let $a_{2}$ be such a vertex. If $G$ contains a vertex at distance exactly three from $\left\{a_{1}, a_{2}\right\}$ then let $a_{3}$ be such a vertex. If $G$ contains a vertex at distance exactly three from $\left\{a_{1}, a_{2}, a_{3}\right\}$ then let $a_{4}$ be such a vertex. We repeat this step until there is no vertex at distance three from, say, $\left\{a_{1}, a_{2}, \ldots, a_{\ell}\right\}$. Hence we obtain a packing $\left\{a_{1}, a_{2}, \ldots, a_{\ell}\right\}=: A$ such that every vertex of $G$ is within distance two of some vertex of $A$.

It follows from the process in which we obtain $A$ that every vertex $a_{i} \in A$ is within distance three of some vertex in $\left\{a_{1}, a_{2}, \ldots, a_{i-1}\right\}$, and so the graph $G^{3}[A]=: H$ is connected. By Lemma 7 we have

$$
\mathcal{E}(H) \leq \mathcal{E}\left(P_{\ell}\right) .
$$

Since in $G$ each vertex of $A$ is within distance at most $\operatorname{3ecc}_{H}\left(a_{i}\right)$ of $a_{i}$, and since each vertex of $G$ is within distance two of some vertex of $A$, we have

$$
\left.\mathcal{E}(G)\right|_{A} \leq 3 \mathcal{E}\left(P_{\ell}\right)+(2)^{\ell} .
$$

For each vertex $a_{i} \in A$ define a set $B_{i}$ of order $\delta+1$ containing $a_{i}$ and exactly $\delta$ of its neighbours, and let $B=\bigcup_{i=1}^{\ell} B_{i}$. For each $v \in B_{i}$ we have $\left|\operatorname{ecc}_{G}(v)-\operatorname{ecc}_{G}\left(a_{i}\right)\right| \leq 1$. Hence, by (12) we get

$$
\left.\mathcal{E}(G)\right|_{B} \leq 3 \mathcal{E}\left(P_{\ell}\right)^{\delta+1}+(3)^{\ell(\delta+1)} .
$$

Now each vertex of $G$ is within distance two of some vertex in $A$, so $\operatorname{diam}(G) \leq 4+\max _{i, j} d_{G}\left(a_{i}, a_{j}\right)=3 \operatorname{diam}(H)+4 \leq 3 \ell+1$. Hence the $n-\ell(\delta+1)$ vertices not in $B$ have eccentricity at most $3 \ell+1$. It follows that

$$
\begin{aligned}
\mathcal{E}(G) & \leq\left.(3 \ell+1)^{n-\ell(\delta+1)} \circ \mathcal{E}(G)\right|_{B} \\
& \leq(3 \ell+1)^{n-\ell(\delta+1)} \circ\left(3 \mathcal{E}\left(P_{\ell}\right)^{\delta+1}+(3)^{\ell(\delta+1)}\right) .
\end{aligned}
$$

Making use of (9) we can thus express the right hand side of the last inequality in terms of $\mathcal{E}\left(G_{n, \delta}^{(\ell)}\right)$ as follows.

$$
\mathcal{E}(G) \leq \mathcal{E}\left(G_{n, \delta}^{(\ell)}\right)+(3)^{n} .
$$


By (10) we thus conclude that

$$
\mathcal{E}(G) \leq \mathcal{E}\left(G_{n, \delta}\right)+(3)^{n},
$$

as desired.

Proposition $2(a)$. Let $G$ be a tree of order $n$, where $n \geq 2$. Then

$$
\mathcal{E}(G) \geq(2)^{n-1} \circ(1)^{1} .
$$

(b) Let $G$ be a planar graph of order $n$, where $n \geq 6$. Then

$$
\mathcal{E}(G) \geq(2)^{n-2} \circ(1)^{2} .
$$

(c) Let $G$ be an outerplanar graph of order $n$, where $n \geq 5$. Then

$$
\mathcal{E}(G) \geq(2)^{n-1} \circ(1)^{1} .
$$

(d) Let $G$ be a connected graph of order $n$ and size $m$. Then

$$
\mathcal{E}(G) \geq(2)^{n-a} \circ(1)^{a},
$$

where $a=\left\lfloor\frac{2 n-1-\sqrt{(2 n-1)^{2}-8 m}}{2}\right\rfloor$.

Equality holds in (a) for $K_{1, n-1}$, in (b) for $K_{2}+P_{n-2}$, in (c) for $K_{1}+P_{n-1}$, and in (d) for the graph $G_{n, m}$, defined as the graph obtained from $K_{a}+\overline{K_{n-a}}$ by adding edges until the graph has $m$ edges.

Proof. Clearly, if $G$ has at most $k$ vertices of eccentricity 1 , then

$$
\mathcal{E}(G) \geq(2)^{n-k} \circ(1)^{k} \text {. }
$$

Then, if $G$ is a tree, it has at most one vertex of eccentricity 1 , so (a) follows. Now, assume that $G$ is planar. To prove (b) it suffices to show that $G$ has at most two vertices of eccentricity 1 . Indeed, otherwise, if $G$ has three vertices of eccentricity one, these vertices together with any three other vertices would induce a subgraph containing $K_{3,3}$, a contradiction to $G$ being planar. Lastly, if $G$ is outerplanar, it has at most one vertex of eccentricity 1 . Otherwise, if $G$ has two vertices of eccentricity one, these vertices together with any three other vertices would induce a subgraph containing $K_{2,3}$, a contradiction to $G$ being outerplanar, and (c) follows. To prove (d) it suffices to show that $G$ contains at most $a$ vertices of degree $n-1$. If $G$ contains $k$ vertices of degree $n-1$, then $G$ contains a spanning subgraph $K_{k}+\overline{K_{n-k}}$, and so $m \geq\left(\begin{array}{l}k \\ 2\end{array}\right)+k(n-k)$. It is easy to verify that $a$ is the largest value of $k$ satisfying this inequality.

\section{Bounds on the average eccentricity of the strong product of graphs}

In this section we consider the average eccentricity of strong products. In our proofs we make extensive use of the results on the eccentric sequence in Section 6. In order to avoid fractions, we consider in our proofs the total eccentricity $\zeta(G)$, defined as the sum of the eccentricities of all vertices of $G$, rather than the average eccentricity. The first result provides a link between eccentric sequences and the average eccentricity of the strong product of graphs.

Lemma 10. Let $G$ and $G^{\prime}$ be two connected graphs of the same order $n_{G}$, and let $H$ be a connected graph.

(a) If $\mathcal{E}(G) \geq \mathcal{E}\left(G^{\prime}\right)$ then

$$
\operatorname{avec}(G \otimes H) \geq \operatorname{avec}\left(G^{\prime} \otimes H\right) \text {. }
$$

(b) If $\mathcal{E}(G)+(c)^{n_{G}} \geq \mathcal{E}\left(G^{\prime}\right)$ where $c \in \mathbb{R}$, then

$$
\operatorname{avec}(G \otimes H)+c \geq \operatorname{avec}\left(G^{\prime} \otimes H\right) \text {. }
$$

Proof. Let $n_{G}$ be the order of $G$ and $G^{\prime}$, and let $n_{H}$ be the order of $H$. Let $\mathcal{E}(G)=\left(e_{1}, e_{2}, \ldots, e_{n_{G}}\right)$ and $\mathcal{E}\left(G^{\prime}\right)=\left(e_{1}^{\prime}, e_{2}^{\prime}, \ldots, e_{n_{G}}^{\prime}\right)$.

(a) It suffices to prove the equivalent statement

$$
\zeta(G \otimes H) \geq \zeta\left(G^{\prime} \otimes H\right)
$$

We make use of the fact that $\operatorname{ecc}_{G \otimes H}((a, u))=\max \left\{\operatorname{ecc}_{G}(a), \operatorname{ecc}_{H}(u)\right\}$ for all $a \in V(G)$ and $u \in V(H)$. Since $\mathcal{E}(G) \geq \mathcal{E}\left(G^{\prime}\right)$ we have $e_{i} \geq e_{i}^{\prime}$ for $i=1,2, \ldots, n_{G}$. Hence

$$
\begin{aligned}
\zeta(G \otimes H) & =\sum_{a \in V(G)} \sum_{u \in V(H)} \operatorname{ecc}_{G \otimes H}(a, u) \\
& =\sum_{a \in V(G)} \sum_{u \in V(H)} \max \left(\operatorname{ecc}_{G}(a), \operatorname{ecc}_{H}(u)\right)
\end{aligned}
$$




$$
\begin{aligned}
& =\sum_{i=1}^{n_{G}} \sum_{u \in V(H)} \max \left(e_{i}, \operatorname{ecc}_{H}(u)\right) \\
& \geq \sum_{i=1}^{n_{G}} \sum_{u \in V(H)} \max \left(e_{i}^{\prime}, \operatorname{ecc}_{H}(u)\right) \\
& =\sum_{a \in V\left(G^{\prime}\right)} \sum_{u \in V(H)} \max \left(\operatorname{ecc}_{G^{\prime}}(a), \operatorname{ecc}_{H}(u)\right) \\
& =\sum_{a \in V\left(G^{\prime}\right)} \sum_{u \in V(H)} \operatorname{ecc}_{G^{\prime} \otimes H}(a, u) \\
& =\zeta\left(G^{\prime} \otimes H\right),
\end{aligned}
$$

as desired.

(b) It suffices to prove the equivalent statement

$$
\zeta(G \otimes H)+c n_{G} n_{H} \geq \zeta\left(G^{\prime} \otimes H\right) .
$$

Since $\mathcal{E}(G)+(c)^{n_{G}} \geq \mathcal{E}\left(G^{\prime}\right)$ we have $e_{i}+c \geq e_{i}^{\prime}$ for $i=1,2, \ldots, n_{G}$, and so, as in the proof of (a),

$$
\begin{aligned}
\zeta(G \otimes H)+c n_{G} n_{H} & =\sum_{a \in V(G)} \sum_{u \in V(H)}\left\{\max \left(\operatorname{ecc}_{G}(a), \operatorname{ecc}_{H}(u)\right)+c\right\} \\
& =\sum_{i=1}^{n_{G}} \sum_{u \in V(H)}\left\{\max \left(e_{i}, \operatorname{ecc}_{H}(u)\right)+c\right\} \\
& \geq \sum_{i=1}^{n_{G}} \sum_{u \in V(H)} \max \left(e_{i}+c, \operatorname{ecc}_{H}(u)\right) \\
& \geq \sum_{i=1}^{n_{G}} \sum_{u \in V(H)} \max \left(e_{i}^{\prime}, \operatorname{ecc}_{H}(u)\right) \\
& =\zeta\left(G^{\prime} \otimes H\right),
\end{aligned}
$$

as desired.

Lemma 10 in conjunction with Lemmas 7-9 and Proposition 2 imply the following theorems.

Theorem 15. Let $H$ be a connected graph. If $G$ is a connected graph of order $n$, then $\operatorname{avec}(G \otimes H) \leq \operatorname{avec}\left(P_{n} \otimes H\right)$.

Setting $H=K_{1}$ yields the following.

Corollary 4 ([6]). Let $G$ be a connected graph of order $n$. Then

$$
\operatorname{avec}(G) \leq \operatorname{avec}\left(P_{n}\right) .
$$

Lemma 8 in conjunction with Lemma 10 yields the following.

Theorem 16. Let $H$ be a connected graph. If $G$ is a $2 r$-connected graph of order $n$ where $r \in \mathbb{N}$, then $\operatorname{avec}(G \otimes H) \leq \operatorname{avec}\left(C_{n}^{r} \otimes H\right)$.

In [6] it was shown that for every graph of order $n$ and minimum degree $\delta$,

$$
\operatorname{avec}(G) \leq \frac{9 n}{4 \delta+4}+\frac{15}{4} \text {. }
$$

This is generalised by the following theorem.

Theorem 17. Let $H$ be a connected graph. If $G$ is a connected graph of order $n$ and minimum degree $\delta$, then $\operatorname{avec}(G \otimes H) \leq \operatorname{avec}\left(G_{n, \delta} \otimes H\right)+3$.

Proposition 2 in conjunction with Lemma 10 yields the following proposition. 
Proposition 3. Let $G$ and $H$ be connected graphs, $n$ and $m$ the order and the size of $G$. Then,

(a) avec $(G \otimes H) \geq \operatorname{avec}\left(K_{1, n-1} \otimes H\right)$.

(b) $\operatorname{avec}(G \otimes H) \geq \operatorname{avec}\left(\left(K_{2}+P_{n-2}\right) \otimes H\right)$.

(c) $\operatorname{avec}(G \otimes H) \geq \operatorname{avec}\left(\left(K_{1}+P_{n-1}\right) \otimes H\right)$.

(d) avec $(G \otimes H) \geq \operatorname{avec}\left(G_{n, m} \otimes H\right)$, being $G_{n, m}$ the graph obtained from $K_{a}+\overline{K_{n-a}}$ by adding edges until the graph has $m$ edges.

\section{Corollary 5 ([29]). Let $G$ be a connected graph of order $n$ and size $m$. Then}

$$
\operatorname{avec}(G) \geq 2-\frac{a}{n},
$$

where $a=\left\lfloor\frac{2 n-1-\sqrt{(2 n-1)^{2}-8 m}}{2}\right\rfloor$.

\section{References}

[1] A. Abiad, B. Brimkov, A. Erey, L. Leshock, X. Martinez-Rivera, O. Suil, S.Y. Song, J. Williford, On the Wiener index, distance cospectrality and transmissionregular graphs, Discrete Appl. Math. 230 (2017) 1-10.

[2] F. Buckley, L. Superville, Distance distributions and mean distance problems, in: Proceedings of the Third Caribbean Conference on Cmbinatorics and Computing, Bridgetown 1981, 67-76, University of the West Indies, Barbados, 1981

[3] R.M. Casablanca, O. Favaron, M. Kouider, Average distance in the strong product of graphs, Util. Math. 94 (2014) 31-48.

[4] P. Dankelmann, On the distance distribution of trees, Discrete Appl. Math. 159 (2011) 945-952. vol. 10.

[5] P. Dankelmann, D. Erwin, W.D. Goddard, S. Mukwembi, H.C. Swart, A characterisation of eccentric sequences of maximal outerplanar graphs, Australas. J. Combin. 58 (3) (2014) 376-391.

[6] P. Dankelmann, W.D. Goddard, C.S. Swart, The average eccentricity of a graph and its subgraphs, Util. Math. 65 (2004) 41-52.

[7] N. De, S.M.A. Nayeem, A. Pal, Total eccentricity index of the hierarchical product of graphs, Int. J. Appl. Comput. Math. 1 (3) (2015) $503-511$.

[8] M. Dehmer, Y. Shi, A. Mowshowitz, Discrimination power of graph measures based on complex zeros of the partial Hosoya polynomial, Appl. Math. Comput. 250 (2015) 352-355.

[9] E. Deutsch, J.A. Rodriguez-Velazquez, The Hosoya polynomial of distance-regular graphs, Discrete Appl. Math. 178 (2014) $153-156$.

[10] J.K. Doyle, J.E. Graver, Mean distance in a graph, Discrete Math. 17 (1977) 147-154.

[11] R.C. Entringer, D.E. Jackson, D.A. Snyder, Distance in graphs, Czechoslovak Math. J. 26 (1976) 283-296.

[12] K. Fathalikhani, H. Faramarzi, H. Yousefi-Azari, Total eccentricity of some graph operations, Electron. Notes Discrete Math. 45 (2014) 125-131.

[13] O. Favaron, M. Kouider, M. Mahéo, Edge-vulnerability and mean distance, Networks 19 (1989) 493-504.

[14] H. Guo, B. Zhou, H. Lin, The Wiener index of uniform hypergraphs, MATCH Commun. Math. Comput. Chem. 78 (2017) 133-152.

[15] I. Gutman, L. Šoltés, The range of the Wiener index and its mean isomer degeneracy, Z. Naturforsch. A 46 (10) (1991) 865-868.

[16] R. Hammack, W. Imrich, S. Klavžar, Handbook of Product Graphs, second ed, CRC Press, Boca Raton (FL), 2011.

[17] F. Harary, The maximum connectivity of a graph, Proc. Natl. Acad. Sci. 48 (1962) 1142-1146.

[18] H. Hosoya, On some counting polynomials in chemistry, Discrete Appl. Math. 19 (1988) 239-257.

[19] H. Hua, B. Ning, Wiener index, Harary index and hamiltonicity of graphs, MATCH Commun. Math. Comput. Chem. 78 (2017) 153-162.

[20] M. Knor, S. Majstorović, R. Škrekovski, Graphs whose Wiener index does not change when a specific vertex is removed, Discrete Appl. Math. 238 (2018) $126-132$.

[21] L. Lesniak, Eccentric sequences in graphs, Period. Math. Hungar. 6 (4)(1975) 287-293.

[22] L. Lovász, Combinatorial Problems and Exercises, Akadémiai Kiadó, Budapest, 1979.

[23] I. Peterin, P. Pleteršek, The Wiener index of strong product of graphs, Opuscula Math. 38 (1) (2018) 81-94.

[24] J. Plesník, On the sum of all distances in a graph or digraph, J. Graph Theory 8 (1984) 1-24.

[25] D.H. Rouvray, The rich legacy of half century of the Wiener index, in: D.H. Rouvray, R.B. King (Eds.), Topology in Chemistry - Discrete Mathematics of Molecules, Horwood, Chichester, 2002, pp. 16-37.

[26] B.E. Sagan, Y.-N. Yeh, P. Zhang, The Wiener polynomial of a graph. arXiv preprint math/9801011, 1998.

[27] P. Slater, Counterexample to Randic's conjecture on distance degree sequences for trees, J. Graph Theory 6 (1982) 89-92.

[28] L. Šoltés, Transmission in graphs: A bound and vertex removing, Math. Slovaca 41 (1991) 11-16.

[29] Y. Tang, B. Zhou, On average eccentricity, MATCH Commun. Math. Comput. Chem. 67 (2012) 405-423.

[30] N. Tratnik, P.Žigert Pleteršek, Relationship between the Hosoya polynomial and the edge-Hosoya polynomial of trees, MATCH Commun. Math. Comput. Chem. 78 (2017) 181-187.

[31] H. Wang, A.T. Amin, Realizability of a tree with a given sequence as its partial distance distribution, Congr. Numer. 110 (1995) $193-199$.

[32] Y.-N. Yeh, I. Gutman, On the sum of all distances in composite graphs, Discrete Math. 135 (1994) 359-365. 